\title{
Patents targeting the Warburg effect for cancer therapy: an interview with William P Katt
}

\author{
William P Katt*,1(i) \\ ${ }^{1}$ Department of Molecular Medicine, Cornell University, Ithaca, NY 14853 6401, USA \\ *Author for correspondence: wpk8@cornell.edu
}

\begin{abstract}
"Many of the proteins a cancer depends upon for metabolism are of minimal to no importance for most other cells."
\end{abstract}

Dr William Katt is a multidisciplinary scientist with particular focus in computational, synthetic and biological chemistry. He obtained his undergraduate degree at Rensselaer Polytechnic Institute and performed his graduate studies at Yale University, where he focused on designing small-molecule inhibitors of the Rho/Rho GEF interaction. Following those studies, Dr Katt accepted a fellowship from the American Cancer Society which funded his work at Cornell University, where he investigated small-molecule inhibitors of the enzyme glutaminase, a key player in cancer metabolism. Today, Dr Katt is a research associate at Cornell and maintains a number of collaborations with researchers across the nation examining glutaminase, cancer stem cells, nano-therapeutics and more, with the goal of developing therapeutic approaches that will eventually help patients in the clinic.

First draft submitted: 21 January 2020; Accepted for publication: 23 January 2020; Published online: 14 February 2020

Keywords: cancer • glutaminase $\bullet \mathrm{IDH} 2 \bullet$ interview $\bullet$ metabolism

\section{Can you give us a short summary of your recent article 'Inhibition of cancer metabolism: a patent landscape' [1]?}

Certainly. We give an overview of some of the approaches being taken to develop anticancer therapeutics by targeting metabolic pathways. Of course, 'cancer metabolism' is too broad a topic to hope to examine in a single article and so we limited ourselves to the Warburg effect: the metabolic changes that cancer cells undergo relating primarily to glycolysis, glutaminolysis and the tricarboxylic acid cycle. In the end, we discuss 60-70 different patents, describing about 50 specific small molecules to show what scaffolds are currently being examined.

\section{What role does the Warburg effect play in the pathogenesis of cancer?}

Great question. For most cancer cells, the Warburg effect goes hand-in-hand with tumorigenesis. The Warburg effect, at its simplest, is a series of metabolic changes that are encountered in cells trying to proliferate rapidly. For any given cell, input nutrients are used for two primary purposes: the generation of energy (e.g., the so-called high-energy molecules such as ATP) and the production of biochemical 'building blocks' to make more DNA, RNA, or protein. Your average slowly proliferating cell tends to prioritize energy generation and tries to get every last ounce of energy out of its nutrients, which means not much carbon from those input molecules gets used for building blocks. For a cancer, though, the goal is to proliferate rapidly, so building block production becomes prioritized and metabolic pathways adapt to this alternate use of input nutrients. What is really interesting to me is that if you alter a healthy cell to mimic those cancer metabolic pathways, not much happens; but without those changes, a tumor can not grow. The changes are not causative, but they are necessary in a tremendous number of cases. 


\section{Why is cancer metabolism such a good target for pharmaceutical intervention?}

I think the biggest reason is that the metabolism is so totally different from the average healthy cell. Many of the proteins a cancer depends upon for metabolism are of minimal to no importance for most other cells. This offers a potentially wider therapeutic window, especially compared with traditional chemotherapeutics such as cisplatin, which damage the DNA in all cells and you just hope the cancer dies first.

\section{Cancer metabolism can be targeted via several different pathways (i.e., glycolysis, anaplerosis, the TCA cycle \& nutrient import). In your opinion, which of these holds the most promise for cancer therapy in terms of their patent landscape?}

From a personal point of view, I think glutaminase holds a lot of promise. It helps feed the tricarboxylic acid cycle when glucose is being diverted into building block production. Inhibiting glutaminase is a major focus of my own research and inhibitors slow the growth of a wide variety of cancer cells; but honestly, I think the greatest benefit in the next decade is going to come from mutant IDH2, part of the TCA cycle. Mutant IDH2 plays a major role in supporting the growth of some cancers. It is not as broadly applicable a target as glutaminase, but there has been much more work done on mutant IDH2 inhibitors, with many different chemical scaffolds having been investigated. One such compound is already in the clinic helping people, and more are surely on the way.

\section{Are there any highlights for patents which target this pathway? What makes these stand out?}

I would say the highlights from me are the filings from Agios - they have historically been one of the leaders in mutant IDH2 inhibitor research and scaffolds they design often set trends. In fact, their drug enasidenib has advanced to the clinic, having gained US FDA approval in August 2017. So I would say they are the ones to watch for future developments.

\section{How would you like to see this field develop in the future?}

I would really like to see more research done on under-investigated targets. It can be hard to justify the investment, when mutant IDH2 has already had some clinical success and glutaminase is so well under waym, but there is a lot of untapped potential in the enzymes that have only been minimally investigated, or not studied at all. The more different targets we have and the more inhibitors we have for each target, the more likely we'll have the right tool to help any given patient overcome their cancer.

\section{Disclaimer}

The opinions expressed in this interview are those of the interviewee and do not necessarily reflect the views of Future Science Ltd.

Acknowledgments

Thanks go to Dr. Michael Lukey, for many valuable discussions pertaining to cancer metabolism over the years.

\section{Financial \& competing interests disclosure}

Dr Katt is a listed coauthor on patents relating to the inhibition of the enzyme glutaminase. This interview discusses work funded in part by grants from the NIH to Richard A Cerione (GM12575 and CA201402). The author has no other relevant affiliations or financial involvement with any organization or entity with a financial interest in or financial conflict with the subject matter or materials discussed in the manuscript apart from those disclosed.

No writing assistance was utilized in the production of this manuscript.

\section{Reference}

1. Katt WP, Cerione RA. Inhibition of cancer metabolism: a patent landscape. Pharm. Pat. Anal. 8(4), 117-138 (2019). 